Portland State University

PDXScholar

10-1-1972

\title{
Existence of the Dielectric Constant in Rigid-Dipole Fluids: The Direct Correlation Function
}

John D. Ramshaw

Portland State University, jdramshaw@yahoo.com

Follow this and additional works at: https://pdxscholar.library.pdx.edu/phy_fac

Part of the Physics Commons

Let us know how access to this document benefits you.

\section{Citation Details}

J.D. Ramshaw, "Existence of the Dielectric Constant in Rigid-Dipole Fluids: The Direct Correlation Function," J. Chem. Phys. 57, 2684 (1972)

This Article is brought to you for free and open access. It has been accepted for inclusion in Physics Faculty Publications and Presentations by an authorized administrator of PDXScholar. Please contact us if we can make this document more accessible: pdxscholar@pdx.edu. 


\title{
Existence of the Dielectric Constant in Rigid-Dipole Fluids: The Direct Correlation Function
}

\author{
JoHN D. RAMSHAw* \\ Department of Physics, The University of Utah, Salt Lake City, Utah 84112
}

(Received 10 January 1972)

\begin{abstract}
The question of whether the dielectric constant $\epsilon$ exists (is well defined) for a finite fluid system of rigid dipolar molecules is reconsidered and reformulated. It is found that this question can most simply be expressed in terms of the behavior of the position- and orientation-dependent direct correlation function $c\left(\mathbf{r}_{1}, \omega_{1} ; \mathbf{r}_{2}, \omega_{2}\right)$. It is shown that $\epsilon$ exists if $c$ satisfies the following two conditions: (a) $c \sim-\phi / k T$ for $\left|\mathbf{r}_{1}-\mathbf{r}_{2}\right|>\sigma$, where $\phi$ is the dipole-dipole potential and $\sigma$ is a length which is large microscopically but small macroscopically. (b) $c\left(\mathbf{r}_{1}, \boldsymbol{\omega}_{1} ; \mathbf{r}_{2}, \boldsymbol{\omega}_{2}\right)$ is of the form $c_{s}\left(\left|\mathbf{r}_{1}-\mathbf{r}_{2}\right|\right)+\mathbf{F}\left(\mathbf{r}_{1}-\mathbf{r}_{2}\right): e\left(\boldsymbol{\omega}_{1}\right) \mathbf{e}\left(\boldsymbol{\omega}_{2}\right)$ for $\left|r_{1}-r_{2}\right|<\sigma$, where $e(\omega)$ is the unit vector with orientation $\omega$. An explicit (and new) expression for $\epsilon$ in terms of $c$ is automatically obtained; its applicability is ensured if the above conditions are satisfied. These results lend new intuition and insight into the question of the existence of $\epsilon$, and suggest a promising approach for future investigations of this question.
\end{abstract}

\section{INTRODUCTION}

The dielectric constant $\epsilon$ exists (is well defined) only if there exists a constant $\chi$, independent of position and sample geometry, such that $\mathbf{P}(\mathbf{r})=\chi \mathbf{E}(\mathbf{r})$, where $\mathbf{P}(\mathbf{r})$ and $\mathbf{E}(\mathbf{r})$ are, respectively, the polarization (dipole moment per unit volume) and the macroscopic Maxwell electric field at the point $\mathbf{r}$ within the dielectric. If such a constant exists, the dielectric constant $\epsilon$ is defined in terms of it by the equation $\epsilon=1+4 \pi \chi$. Since $\mathbf{P}(\mathbf{r})$ and $\mathbf{E}(\mathbf{r})$ are well defined regardless of whether $\epsilon$ exists, the existence of $\epsilon$ cannot be guaranteed by definition, but must be established by theory or experiment. This point has been discussed in greater detail elsewhere. ${ }^{1-3}$

Most theoretical work on dielectrics has been conducted within the framework of the assumption that $\epsilon$ exists. ${ }^{4}$ This assumption enables one to derive a molecular expression for $\epsilon$, but the conditions under which this expression is applicable are not revealed by the derivation. The question of whether $\epsilon$ in fact exists has only recently begun to receive much attention. The first systematic investigation of this question was apparently that of Kaufman and Watson. ${ }^{5}$ They develop a very general quantum-mechanical approach which is applicable to both polar and nonpolar dielectrics. A microscopic expression for $\mathbf{P}(\mathbf{r})$ is obtained by means of a linked-diagram expansion of the partition function in the presence of the external field. The dielectric constant is then found to exist if certain graphs in the expansion are neglected, in which case it is obtained as a power series in the activity. Because of the activity expansion, these results apply only to gases.

The approach of Kaufman and Watson is so general that the role of intermolecular correlations is difficult to extract from the theory. Other investigations have focused attention directly upon the relation between such correlations and the existence of $\epsilon$. For gases or liquids composed of nonpolar molecules with densityindependent harmonic polarizabilities, it has recently been demonstrated that $\epsilon$ exists at least to third order in the polarizability. ${ }^{3}$ This result is obtained in spite of (and as a consequence of) the fact that the intermolecular correlations in such fluids are expected to be purely short ranged in nature. In polar fluids, however, the situation is quite different (and perhaps more interesting) due to the long-range nature of the permanent dipole-dipole interaction. In fact, the existence of $\epsilon$ in polar fluids is found to be intimately connected with the existence and nature of long-range orientational intermolecular correlations in the unperturbed fluid., ${ }^{\mathbf{1} 2}$ This connection was established and discussed in a previous publication, ${ }^{2}$ in which we restricted attention to rigid (unpolarizable) polar molecules in order to avoid inessential complications. We obtained the following rigorous expression for $\mathbf{P}(\mathbf{r})$ :

$$
\mathbf{P}(\mathbf{r})=\int_{\boldsymbol{V}} d^{3} \mathbf{r}^{\prime} \mathbf{K}\left(\mathbf{r}, \mathbf{r}^{\prime}\right) \cdot \mathbf{E}_{\mathbf{0}}\left(\mathbf{r}^{\prime}\right),
$$

where the integration is extended over the volume $V$ (whose shape is left arbitrary) occupied by the sample, and $\mathbf{E}_{0}(\mathbf{r})$ is the externally applied electric field. The dyadic kernel $\mathbf{K}\left(\mathbf{r}, \mathbf{r}^{\prime}\right)$ is given by

$$
\begin{aligned}
\mathbf{K}\left(\mathbf{r}, \mathbf{r}^{\prime}\right)= & \mu_{0}^{2} \beta\left[\frac{\mathbf{1}}{3} \rho \mathbf{U} \delta\left(\mathbf{r}-\mathbf{r}^{\prime}\right)\right. \\
& \left.+\int d \boldsymbol{\omega}_{1} d \boldsymbol{\omega}_{2} \rho^{(2)}\left(\mathbf{r}, \boldsymbol{\omega}_{1} ; \mathbf{r}^{\prime}, \boldsymbol{\omega}_{2}\right) \mathbf{e}\left(\boldsymbol{\omega}_{1}\right) \mathbf{e}\left(\boldsymbol{\omega}_{2}\right)\right]
\end{aligned}
$$

where $\mu_{0}$ is the magnitude of the permanent molecular dipole moment, $\beta=(k T)^{-1}, \rho$ is the number density, $U$ is the unit dyadic, $\omega_{k}$ denotes the set of Euler angles which specify the orientation of molecule $k$ relative to the laboratory coordinate system, $\mathbf{e}(\boldsymbol{\omega})$ is the unit vector with orientation $\omega$, and $\rho^{(2)}$ is the position- and orientation-dependent two-molecule distribution function. For simplicity the molecules are taken to be axially symmetric, so that $\omega_{k}=\left(\theta_{k}, \phi_{k}\right)$ and $d \omega_{k}=$ $\sin \theta_{k} d \theta_{k} d \phi_{k}$, where $\theta_{k}$ and $\phi_{k}$ are the usual azimuthal and polar angles.

Equations (1) and (2) form the basis for the connection between the existence of $\epsilon$ and the nature of the intermolecular correlations. If $\epsilon$ exists then $\mathbf{P}(\mathbf{r})$ is locally related to $\mathbf{E}(\mathbf{r})$, which requires that the relation 
between $\mathbf{P}(\mathbf{r})$ and the external field $\mathbf{E}_{0}(\mathbf{r})$ be nonlocal. If this is the case, then according to Eqs. (1) and (2) the pair distribution function $\rho^{(2)}$ must contain orientational correlations of a long-range nature. In order to investigate the existence of $\epsilon$, it then becomes necessary to evaluate the long-range part of $\rho^{(2)}$ to see whether its behavior is such as to imply [via Eqs. (1) and (2)] a local relation between $\mathbf{P}(\mathbf{r})$ and $\mathbf{E}(\mathbf{r})$. We did not attempt to evaluate $\rho^{(2)}$ in general, but by means of a density expansion we were able to show that $\epsilon$ exists at least to second order in $\rho$ for rigid dipolar molecules, provided that the external field $E_{0}(\mathbf{r})$ varies slowly with $\mathbf{r}$ in a molecular sense.

The present article is a continuation and extension of the basic foundation laid in Ref. 2. Here we go on to investigate in general the behavior which $\rho^{(2)}$ must exhibit in order for $\epsilon$ to exist. We find that this investigation is considerably simplified by defining a kernel $\mathbf{L}\left(\mathbf{r}, \mathbf{r}^{\prime}\right)$ which is the inverse of the kernel $K\left(r, r^{\prime}\right)$. If $\epsilon$ exists the quantity $L\left(\mathbf{r}, \mathbf{r}^{\prime}\right)$ has a simple and intuitive form, whereas the form of $\mathbf{K}\left(\mathbf{r}, \mathbf{r}^{\prime}\right)$ is more complicated. The relation between the parts of $L\left(r, r^{\prime}\right)$ and $K\left(r, r^{\prime}\right)$ which depend upon molecular correlations is found to be of essentially the same form as the well-known relation between the direct and total correlation functions. This suggests that $\mathbf{L}\left(\mathbf{r}, \mathbf{r}^{\prime}\right)$ may be simply related to the direct correlation function in much the same way that $K\left(r, r^{\prime}\right)$ is related to $\rho^{(2)}$. We are therefore led to expect that it may be advantageous to focus attention on the direct correlation function rather than on $\rho^{(2)}$. In order to pursue this idea, it is necessary first to generalize the concept of the direct correlation function to a finite fluid system composed of rigid polar molecules. This generalization is trivial; it is merely necessary to allow for the presence of the orientational degrees of freedom, and for the fact that $\rho^{(2)}\left(\mathbf{r}, \boldsymbol{\omega}_{1} ; \mathbf{r}^{\prime}, \boldsymbol{\omega}_{2}\right)$ depends separately upon the position vectors $\mathbf{r}$ and $\mathbf{r}^{\prime}$ rather than simply upon $\left(\mathbf{r}-\mathbf{r}^{\prime}\right)$.

This approach proves to be a fruitful one. We find that the following two conditions on the direct correlation function are sufficient to guarantee the existence of $\epsilon$ [provided, as usual, that $\mathrm{E}_{\mathbf{0}}(\mathbf{r})$ varies slowly in a molecular sense]: (a) The direct correlation function becomes asymptotically equal to $-\beta \phi$ for $\left|\mathrm{r}-\mathrm{r}^{\prime}\right|>\sigma$, where $\phi$ is the intermolecular pair potential and $\sigma$ is a length which is small macroscopically but much larger than molecular dimensions. For rigid polar molecules $\phi$ differs negligibly from the dipole-dipole potential $\phi_{d}$ if $\left|\mathbf{r}-\mathbf{r}^{\prime}\right|>\sigma$, so that $\phi$ may be replaced by $\phi_{d}$ in the above statement. (b) For $\left|\mathbf{r}-\mathbf{r}^{\prime}\right|<\sigma$, the direct correlation function is of the form

$$
c_{s}\left(\left|\mathbf{r}-\mathbf{r}^{\prime}\right|\right)+\mathbf{F}\left(\mathbf{r}-\mathbf{r}^{\prime}\right): \mathbf{e}\left(\boldsymbol{\omega}_{1}\right) \mathbf{e}\left(\boldsymbol{\omega}_{2}\right),
$$

where, of course, $c_{s}(|\mathbf{r}|)$ and $\mathbf{F}(\mathbf{r})$ remain finite as $|\mathbf{r}| \rightarrow 0$. The existence of $\epsilon$ is rigorously implied by these conditions, and an explicit expression for it in terms of the direct correlation function is automatically obtained. To our knowledge, this expression has not been derived previously.

Whether conditions (a) and (b) are actually satisfied will not be investigated in the present article. However, a few comments pertinent to this question are in order. Condition (a) is frequently said to be satisfied, far from the critical point, for fluids composed of molecules with short-range intermolecular potentials. ${ }^{6}$ Its validity for polar fluids as well has also been claimed ${ }^{7}$ and does not seem unlikely, although to our knowledge no rigorous proof is available. Condition (b) is almost certainly not rigorously satisfied, for in essence it requires the orientation-dependent term in the short-range part of the direct correlation function to have the same angular symmetry as the dipole-dipole potential. It is therefore likely that condition (b) is valid only to some unknown degree of approximation. The quality of this approximation will depend upon the context; It may conceivably be high for dielectric polarization but low in other contexts.

The existence of $\epsilon$ for rigid-dipole fluids has also been investigated by Nienhuis and Deutch. ${ }^{8}$ These authors attempt the direct evaluation of $\rho^{(2)}$ by resumming a density expansion. A graphical representation is utilized which is specifically suited to the presence of the longrange dipole-dipole interaction. By making certain approximations, they are able to formally sum the expansion to infinite order. The resulting approximate expression for $\rho^{(2)}$ is then combined with Eqs. (1) and (2) above. Provided that $\mathbf{E}_{0}(\mathbf{r})$ varies slowly in a molecular sense, it is found that $\mathbf{P}(\mathbf{r})$ is indeed locally proportional to $\mathbf{E}(\mathbf{r})$, so that $\epsilon$ exists under the conditions of the derivation.

Nienhuis and Deutch state that their results demonstrate the existence of $\epsilon$ for rigid-dipole fluids "apart from some minor and completely acceptable restrictions." This is perhaps too strong a statement, for their derivation contains several assumptions and approximations whose cumulative effect is difficult to assess. In particular, in obtaining their Eq. (3.29) from their Eq. (3.28) they make an approximation whose quality is neither explored nor estimated, It is also noteworthy that their derivation is based upon a density expansion, a fact which implies that its results are strictly applicable only to gases. It is likely, however, that the same results could be obtained (from the same approximations) by functional derivative techniques, ${ }^{9}$ which are not subject to this limitation.

Thus, while the approach of Nienhuis and Deutch is not without interest, their results can in no sense be regarded as conclusive. We feel that the formulation developed in the present article is to be preferred because of its considerably greater simplicity and intuitive appeal. By transforming the problem so that the direct correlation function becomes the object of interest, we find that conditions sufficient to guarantee the existence of $\epsilon$ can be expressed very simply indeed. 
Condition (a) above, in particular, appeals strongly to intuition and to what we know about the behavior of nonpolar fluids. If this condition is satisfied than it is the convolution in the equation relating the direct correlation function to $\rho^{(2)}$ which provides the mechanism by which the simple behavior of the direct correlation function is transformed into a complex shapedependent behavior for $\boldsymbol{\rho}^{(2)}$. Our results therefore provide new intuition and insight into the question of the existence of $\epsilon$ for rigid-dipole fluids, and suggest that future investigations of this question may expect to profitably restrict attention to the direct correlation function, and in particular to investigating the validity of conditions (a) and (b) above.

The new expression for $\epsilon$ which emerges from our treatment bears no apparent resemblance to the Kirkwood equation, which emerges from the treatment of Nienhuis and Deutch. Within the framework of conditions (a) and (b), however, these two expressions are in fact equivalent. This follows from the fact that the Kirkwood equation is a logical consequence of the assumption that $\epsilon$ exists, while the existence of $\epsilon$ is a logical consequence of conditions (a) and (b). It would therefore be of interest to know the extent to which the various approximations made by Nienhuis and Deutch are equivalent to conditions (a) and (b) above. One wonders in particular whether the Nienhuis-Deutch graphical-expansion treatment of $\rho^{(2)}$ is not in essence an indirect justification of our condition (a). In this connection, it is noteworthy that our condition (a) corresponds closely to the lowest-order result of the $\gamma$-ordering theory of Lebowitz, Stell, and Baer ${ }^{10}$ [their Eq. (5.18)], and that if one combines these authors' equations (5.7) and (5.18) one obtains a result which, for a polar fluid, appears equivalent to Eq. (3.28) of Nienhuis and Deutch. This correspondence is certainly suggestive, and lends further support to our contention that the direct correlation function provides the simplest and most fundamental approach to the problem.

\section{THE INVERSE KERNEL}

We emphasize again that the present article is a natural extension of Ref. 2, familiarity with which is assumed throughout the following development. Discussions and explanations given in Ref. 2 will not be repeated here; unless otherwise stated, everything in the present article (e.g., the physical situation, the molecular model, the notation, etc.) is the same as in Ref. 2. The basic results of Ref. 2 have already been quoted as Eqs. (1) and (2) above.

If the dielectric constant exists, then

$$
\mathbf{P}(\mathbf{r})=(\epsilon-1) \mathbf{E}(\mathbf{r}) / 4 \pi \quad(\mathbf{r} \text { in } \mathbf{V}),
$$

where $\epsilon$ is a constant of the material, dependent upon density and temperature but independent of position and sample geometry. The field $\mathbf{E}(\mathbf{r})$ is the macroscopic Maxwell electric field to be obtained by solving the
Maxwell equations of electrostatics. It is convenient, however, to eliminate $\mathbf{E}(\mathbf{r})$ in favor of the "Lorentz electric field" $\mathrm{E}_{L}(\mathbf{r})$, which is defined by

$$
\mathbf{E}_{L}(\mathbf{r})=\mathbf{E}(\mathbf{r})+(4 \pi / 3) \mathbf{P}(\mathbf{r}) .
$$

Combining Eqs. (3) and (4), we see that if $\epsilon$ exists then $\mathbf{P}(\mathbf{r})=(3 / 4 \pi)[(\epsilon-1) /(\epsilon+2)] \mathbf{E}_{L}(\mathbf{r}), \quad(\mathbf{r}$ in $\mathbf{V})$.

We use the Lorentz field $\mathbf{E}_{L}(\mathbf{r})$ rather than the Maxwell field $\mathbf{E}(\mathbf{r})$ because $\mathbf{E}_{L}(\mathbf{r})$ is the field within a small spherical cavity at the point $\mathbf{r}$, whereas $\mathbf{E}(\mathbf{r})$ is the field within a small needle-shaped cavity with axis along $\mathbf{P}(\mathbf{r})$. The spherical cavity is mathematically more convenient to deal with. We emphasize that Eq. (4) is simply the definition of $\mathbf{E}_{L}(\mathbf{r})$; we do not assume that $E_{L}(\mathbf{r})$ may be identified with the average local field acting on a representative molecule at $r$, nor does such an average local field play any part in our development. The explicit expression for $\mathbf{E}_{L}(\mathbf{r})$ in terms of $\mathbf{P}(\mathbf{r})$ is

$$
\mathbf{E}_{L}(\mathbf{r})=\mathbf{E}_{0}(\mathbf{r})+\lim _{\delta \rightarrow 0} \int d^{3} \mathbf{r}^{\prime} \mathbf{T}_{\delta}\left(\mathbf{r}-\mathbf{r}^{\prime}\right) \cdot \mathbf{P}\left(\mathbf{r}^{\prime}\right) .
$$

Here $\mathbf{T}_{\delta}(\mathbf{r})=\theta(|\mathbf{r}|-\delta) \mathbf{T}(\mathbf{r})$, where $\mathbf{T}(\mathbf{r})$ is the dipoledipole interaction tensor $\nabla \nabla|\mathbf{r}|^{-1}$ and $\theta(x)$ is the Heaviside unit step function, defined to be unity if $x \geq 0$ and zero otherwise. Equation (6) is equivalent to the Maxwell equations; it is obtained by combining the definition of $\mathbf{E}_{L}(\mathbf{r})$, Eq. (4), with Eq. (1) of Ref. 2.

We now define $\mathbf{L}\left(\mathbf{r}, \mathbf{r}^{\prime}\right)$ to be the inverse of the kernel $K\left(r, r^{\prime}\right)$ which appears in Eq. (1), so that $t^{11}$

$$
\mathbf{E}_{0}(\mathbf{r})=\int_{V} d^{3} \mathbf{r}^{\prime} \mathbf{L}\left(\mathbf{r}, \mathbf{r}^{\prime}\right) \cdot \mathbf{P}\left(\mathbf{r}^{\prime}\right) \quad(\mathbf{r} \text { in } \mathbf{V}) .
$$

By substituting Eq. (7) into Eq. (1), we find that $\mathbf{K}\left(\mathbf{r}, \mathbf{r}^{\prime}\right)$ and $\mathbf{L}\left(\mathbf{r}, \mathbf{r}^{\prime}\right)$ are related by

$$
\int_{V} d^{3} \mathbf{r}_{1} \mathbf{K}\left(\mathbf{r}, \mathbf{r}_{1}\right) \cdot \mathbf{L}\left(\mathbf{r}_{1}, \mathbf{r}^{\prime}\right)=\mathbf{U} \delta\left(\mathbf{r}-\mathbf{r}^{\prime}\right) .
$$

We now proceed to examine the behavior which $L\left(r, r^{\prime}\right)$ must exhibit in order for $\epsilon$ to exist. We first restrict attention to external fields $E_{0}(\mathbf{r})$ which vary slowly with $\mathbf{r}$ in a molecular sense. By this we mean that $\mathbf{E}_{0}(\mathbf{r}+\mathbf{a})$ differs negligibly from $\mathbf{E}_{0}(\mathbf{r})$ if $|\mathbf{a}|<\sigma$, where $\sigma$ is a distance which is small macroscopically but much larger than a molecular diameter. For concreteness we may take $\sigma \approx 1000 \AA=10^{-5} \mathrm{~cm}$. Since our concern is limited to fluids, $\mathbf{P}(\mathbf{r})$ may safely be assumed to vary slowly with $\mathbf{r}$ in the same sense as $E_{0}(\mathbf{r})$. This in turn implies $^{2,3}$ that the integral in Eq. (6) becomes independent of $\delta$ if $\delta \leqq \sigma$. The limit as $\delta \rightarrow 0$ in Eq. (6) may therefore be achieved simply by setting $\delta=\sigma$, so that

$$
\mathrm{E}_{L}(\mathbf{r})=\mathbf{E}_{0}(\mathbf{r})+\int d^{3} \mathbf{r}^{\prime} \mathbf{T}_{\sigma}\left(\mathbf{r}-\mathbf{r}^{\prime}\right) \cdot \mathbf{P}\left(\mathbf{r}^{\prime}\right)
$$

We next eliminate $\mathbf{E}_{0}(\mathbf{r})$ from Eq. (9) by means of Eq. (7), obtaining

$\mathbf{E}_{L}(\mathbf{r})=\int_{V} d^{3} \mathbf{r}^{\prime}\left[\mathbf{L}\left(\mathbf{r}, \mathbf{r}^{\prime}\right)+\mathbf{T}_{\sigma}\left(\mathbf{r}-\mathbf{r}^{\prime}\right)\right] \cdot \mathbf{P}\left(\mathbf{r}^{\prime}\right) \quad(\mathbf{r}$ in $\mathbf{V})$. 
Now in order for $\epsilon$ to exist, Eq. (10) must reduce to the local relation (5) between $\mathbf{P}(\mathbf{r})$ and $\mathbf{E}_{L}(\mathbf{r})$. This requires, first of all, that $\left[\mathrm{L}\left(\mathrm{r}, \mathrm{r}^{\prime}\right)+\mathrm{T}_{\sigma}\left(\mathrm{r}-\mathrm{r}^{\prime}\right)\right]$ be short ranged. That is, $\mathbf{L}\left(\mathbf{r}, \mathbf{r}^{\prime}\right)$ must be of the form

$$
\mathbf{L}\left(\mathbf{r}, \mathbf{r}^{\prime}\right)=\mathrm{L}_{s}\left(\mathbf{r}, \mathbf{r}^{\prime}\right)-\mathbf{T}_{\sigma}\left(\mathbf{r}-\mathbf{r}^{\prime}\right),
$$

where $L_{s}\left(\mathbf{r}, \mathbf{r}^{\prime}\right) \approx 0$ if $\left|\mathbf{r}-\mathbf{r}^{\prime}\right|>\sigma$. If this is the case then Eq. (10) reduces to

$$
\mathbf{E}_{L}(\mathbf{r})=\mathbf{A}(\mathbf{r}) \cdot \mathbf{P}(\mathbf{r}) \quad(\mathbf{r} \text { in } \mathbf{V}),
$$

where

$$
\mathbf{A}(\mathbf{r})=\int_{\infty} d^{3} \mathbf{r}^{\prime} \mathbf{L}_{s}\left(\mathbf{r}, \mathbf{r}^{\prime}\right) .
$$

The " $\infty$ " beneath the integral sign in Eq. (13) indicates that the integration is extended over all space. Although it is local, Eq. (12) does not yet imply the existence of $\epsilon$. We must further require that

$$
\mathrm{A}(\mathrm{r})=A \mathrm{U},
$$

where $A$ is independent of $\mathrm{r}$. Since $\operatorname{Tr} \mathrm{U}=3, A=\frac{1}{3} \operatorname{TrA}(\mathrm{r})$. Combining Eqs. (14) and (12), we obtain

$$
\mathbf{E}_{L}(\mathbf{r})=A \mathbf{P}(\mathbf{r}) \quad(\mathbf{r} \text { in } \mathrm{V}) .
$$

Equation (15) is of the form of Eq. (5), with

$$
\begin{aligned}
(3 / 4 \pi)[(\epsilon-1) /(\epsilon+2)] & =A^{-1} \\
& =\left[\frac{1}{3} \operatorname{Tr} \int_{\infty} d^{3} \mathbf{r}^{\prime} \mathbf{L}_{8}\left(\mathbf{r}, \mathbf{r}^{\prime}\right)\right]^{-1} .
\end{aligned}
$$

We see, therefore, that in order for $\epsilon$ to exist the longrange part of $L\left(r, r^{\prime}\right)$ must simply be equal to $-T_{\sigma}\left(r-r^{\prime}\right)$. In addition, the behavior of the short-range part $\mathbf{L}_{s}\left(\mathbf{r}, \mathbf{r}^{\prime}\right)$ must be such that the quantity $\mathbf{A}(\mathbf{r})$, given by Eq. (13), is both independent of $\mathbf{r}$ and proportional to the unit dyadic $U$. The dielectric constant exists if and only if these conditions are satisfied, in which case it is given by Eq. (16).

It is now advantageous to separate out the parts of $K\left(\mathbf{r}, \mathbf{r}^{\prime}\right)$ and $L\left(\mathbf{r}, \mathbf{r}^{\prime}\right)$ which depend upon the intermolecular correlations. To this end, we define a correlation tensor $\mathbf{G}\left(\mathbf{r}, \mathbf{r}^{\prime}\right)$ by

$\mathbf{G}\left(\mathbf{r}, \mathbf{r}^{\prime}\right)=\rho^{-2} \int d \omega_{1} d \omega_{2} \rho^{(2)}\left(\mathbf{r}, \omega_{1} ; \mathbf{r}^{\prime}, \omega_{2}\right) \mathbf{e}\left(\omega_{1}\right) \mathbf{e}\left(\omega_{2}\right)$,

so that Eq. (2) becomes

$$
\mathbf{K}\left(\mathbf{r}, \mathbf{r}^{\prime}\right)=\mu_{0}^{2} \beta \rho\left[\frac{1}{3} \mathbf{U} \delta\left(\mathbf{r}-\mathbf{r}^{\prime}\right)+\rho \mathbf{G}\left(\mathbf{r}, \mathbf{r}^{\prime}\right)\right] .
$$

Substitution of Eq. (18) into Eq. (8) yields

$$
\mathbf{G}\left(\mathbf{r}, \mathbf{r}^{\prime}\right)=\mathbf{H}\left(\mathbf{r}, \mathbf{r}^{\prime}\right)+3 \rho \int_{V} d^{3} \mathbf{r}_{1} \mathbf{G}\left(\mathbf{r}, \mathbf{r}_{1}\right) \cdot \mathbf{H}\left(\mathbf{r}_{1}, \mathbf{r}^{\prime}\right),
$$

where

$$
\mathbf{H}\left(\mathbf{r}, \mathbf{r}^{\prime}\right)=(3 \rho)^{-1} \mathbf{U} \delta\left(\mathbf{r}-\mathbf{r}^{\prime}\right)-\frac{1}{9} \mu_{0}^{2} \beta \mathbf{L}\left(\mathbf{r}, \mathbf{r}^{\prime}\right) .
$$

According to Eq. (20), we may regard $-\mathbf{H}\left(\mathbf{r}, \mathbf{r}^{\prime}\right)$ as the part of $\left(\mu_{0}^{2} \beta / 9\right) L\left(r, r^{\prime}\right)$ which depends upon the intermolecular correlations.

One now notices that Eq. (19) is of the same form as the well-known Ornstein-Zernike relation ${ }^{9}$ between the direct and total correlation functions, the role of the former being played by $\mathbf{H}\left(\mathbf{r}, \mathbf{r}^{\prime}\right)$ and that of the latter by $\mathbf{G}\left(r, r^{\prime}\right)$. [The factor of 3 in Eq. (19) is due to the tensor character of the equation; its origin is the fact that $\operatorname{Tr} U=3$.] Since $G\left(r, \mathbf{r}^{\prime}\right)$ is simply related by Eq. (17) to the complete pair distribution function $\rho^{(2)}$, we are led to wonder whether $\mathbf{H}\left(\mathbf{r}, \mathbf{r}^{\prime}\right)$ is similarly simply related to the direct correlation function. This question is investigated in the next section.

\section{THE DIRECT CORRELATION FUNCTION}

We must first generalize the concept of the direct correlation function to the case of a finite fluid system of rigid polar molecules. For this purpose, it is convenient to define the totalcorrelation function $h\left(\mathbf{r}, \omega ; \mathbf{r}^{\prime}, \omega^{\prime}\right)$ by the equation,

$$
h\left(\mathbf{r}, \boldsymbol{\omega} ; \mathbf{r}^{\prime}, \boldsymbol{\omega}^{\prime}\right)=(\rho / 4 \pi)^{-2} \rho^{(2)}\left(\mathbf{r}, \boldsymbol{\omega} ; \mathbf{r}^{\prime}, \boldsymbol{\omega}^{\prime}\right)-1 .
$$

In terms of $h\left(\mathbf{r}, \boldsymbol{\omega} ; \mathbf{r}^{\prime}, \boldsymbol{\omega}^{\prime}\right), \mathrm{Eq} . \quad(17)$ for $\mathbf{G}\left(\mathbf{r}, \mathbf{r}^{\prime}\right)$ becomes

$\mathbf{G}\left(\mathbf{r}, \mathbf{r}^{\prime}\right)=(4 \pi)^{-2} \int d \boldsymbol{\omega} d \boldsymbol{\omega}^{\prime} h\left(\mathbf{r}, \boldsymbol{\omega} ; \mathbf{r}^{\prime}, \boldsymbol{\omega}^{\prime}\right) \mathbf{e}(\boldsymbol{\omega}) \mathbf{e}\left(\boldsymbol{\omega}^{\prime}\right)$.

Because we are concerned with a finite (although macroscopic) system, $h\left(\mathbf{r}, \boldsymbol{\omega} ; \mathbf{r}^{\prime}, \boldsymbol{\omega}^{\prime}\right)$ does not strictly approach zero for large $\left|\mathbf{r}-\mathbf{r}^{\prime}\right|$. It approaches instead small terms of order $1 / N$, but since these terms are independent of $\mathbf{r}, \boldsymbol{\omega}, \mathbf{r}^{\prime}$, and $\boldsymbol{\omega}^{\prime}$ they make no contribution to Eq. (22) and may be ignored. For our purposes, therefore, Eq. (21) is an adequate definition of $h\left(\mathbf{r}, \boldsymbol{\omega} ; \mathbf{r}^{\prime}, \boldsymbol{\omega}^{\prime}\right)$, although (as is well known) the $1 / N$ terms may be important in other contexts.

We now define the direct correlation function $c\left(\mathbf{r}, \boldsymbol{\omega} ; \mathbf{r}^{\prime}, \boldsymbol{\omega}^{\prime}\right)$ by the equation,

$h\left(\mathbf{r}, \boldsymbol{\omega} ; \mathbf{r}^{\prime}, \boldsymbol{\omega}^{\prime}\right)=c\left(\mathbf{r}, \boldsymbol{\omega} ; \mathbf{r}^{\prime}, \boldsymbol{\omega}^{\prime}\right)+(\boldsymbol{\rho} / 4 \pi) \int_{V} d^{3} \mathbf{r}_{1} \int d \boldsymbol{\omega}_{1}$

$$
\times h\left(\mathbf{r}, \boldsymbol{\omega} ; \mathbf{r}_{1}, \boldsymbol{\omega}_{1}\right) c\left(\mathbf{r}_{1}, \boldsymbol{\omega}_{1} ; \mathbf{r}^{\prime}, \boldsymbol{\omega}^{\prime}\right) .
$$

Equation (23) is the appropriate generalization of the familiar Ornstein-Zernike equation. ${ }^{9}$ Allowance has been made in Eq. (23) for the presence of the orientational degrees of freedom, for the fact that the system is finite, and for the fact that $h\left(\mathbf{r}, \boldsymbol{\omega} ; \mathbf{r}^{\prime}, \boldsymbol{\omega}^{\prime}\right)$ depends separately upon $\mathbf{r}$ and $\mathbf{r}^{\prime}$ rather than simply upon $\left(\mathbf{r}-\mathbf{r}^{\prime}\right)$. The physical content and intuitive significance of the direct correlation function are in no way diminished by these modifications; they remain the same as in the case of an infinite monatomic fluid with shortrange correlations. Note that Eq. (23) contains a convolution over $\omega_{1}$ as well as $r_{1}$; this angular convolution is essential to the desired physical interpretation of $c\left(\mathbf{r}, \boldsymbol{\omega} ; \mathbf{r}^{\prime}, \boldsymbol{\omega}^{\prime}\right)$.

It is convenient to define a quantity $C\left(r, r^{\prime}\right)$ which is related to the direct correlation function in the same way that $\mathbf{G}\left(r, r^{\prime}\right)$ is related to the total correlation function,

$$
\mathbf{C}\left(\mathbf{r}, \mathbf{r}^{\prime}\right)=(4 \pi)^{-2} \int d \boldsymbol{\omega} d \boldsymbol{\omega}^{\prime} c\left(\mathbf{r}, \boldsymbol{\omega} ; \mathbf{r}^{\prime}, \boldsymbol{\omega}^{\prime}\right) \mathbf{e}(\boldsymbol{\omega}) \mathbf{e}\left(\boldsymbol{\omega}^{\prime}\right) .
$$

In order to investigate the relation between $\mathbf{G}\left(\mathbf{r}, \mathbf{r}^{\prime}\right)$ and $\mathbf{C}\left(\boldsymbol{r}, \mathbf{r}^{\prime}\right)$, we multiply Eq. (23) by the dyad 
$(4 \pi)^{-2} \mathbf{e}(\boldsymbol{\omega}) \mathbf{e}\left(\boldsymbol{\omega}^{\prime}\right)$ and integrate over $\boldsymbol{\omega}$ and $\boldsymbol{\omega}^{\prime}$. The result is

$$
\begin{aligned}
\mathbf{G}\left(\mathbf{r}, \mathbf{r}^{\prime}\right) & =\mathbf{C}\left(\mathbf{r}, \mathbf{r}^{\prime}\right)+(4 \pi)^{-3} \rho \int_{V} d^{3} \mathbf{r}_{1} \int d \boldsymbol{\omega} d \boldsymbol{\omega}_{1} d \boldsymbol{\omega}^{\prime} \\
& \times h\left(\mathbf{r}, \boldsymbol{\omega} ; \mathbf{r}_{1}, \boldsymbol{\omega}_{1}\right) c\left(\mathbf{r}_{1}, \boldsymbol{\omega}_{1} ; \mathbf{r}^{\prime}, \boldsymbol{\omega}^{\prime}\right) \mathbf{e}(\boldsymbol{\omega}) \mathbf{e}\left(\boldsymbol{\omega}^{\prime}\right) .
\end{aligned}
$$

Equation (25) is similar in form to Eq. (19), but the difference is sufficiently great to prevent $H\left(r, r^{\prime}\right)$ from being rigorously equal to $C\left(r, r^{\prime}\right)$ in general. One suspects, however, that $\mathbf{H}\left(\mathbf{r}, \mathbf{r}^{\prime}\right)$ and $\mathbf{C}\left(\mathbf{r}, \mathbf{r}^{\prime}\right)$ may be approximately equal, at least under favorable circumstances. To make this idea precise, let us assume provisionally that $c\left(\mathbf{r}, \boldsymbol{\omega} ; \mathbf{r}^{\prime}, \boldsymbol{\omega}^{\prime}\right)$ is of the form

$$
c\left(\mathbf{r}, \boldsymbol{\omega} ; \mathbf{r}^{\prime}, \boldsymbol{\omega}^{\prime}\right)=c_{s}\left(\mathbf{r}, \mathbf{r}^{\prime}\right)+\mathbf{F}\left(\mathbf{r}, \mathbf{r}^{\prime}\right): \mathbf{e}(\boldsymbol{\omega}) \mathbf{e}\left(\boldsymbol{\omega}^{\prime}\right) .
$$

Substituting Eq. (26) into Eq. (24), we find that

$$
\mathrm{C}\left(\mathrm{r}, \mathbf{r}^{\prime}\right)=\frac{1}{9} \mathrm{~F}\left(\mathrm{r}, \mathbf{r}^{\prime}\right) \text {. }
$$

If we now substitute Eq. (26) into Eq. (25) and make use of Eq. (27), we obtain

$$
\mathbf{G}\left(\mathbf{r}, \mathbf{r}^{\prime}\right)=\mathbf{C}\left(\mathbf{r}, \mathbf{r}^{\prime}\right)+3 \rho \int_{V} d^{3} \mathbf{r}_{1} \mathbf{G}\left(\mathbf{r}, \mathbf{r}_{1}\right) \cdot \mathbf{C}\left(\mathbf{r}_{1}, \mathbf{r}^{\prime}\right) .
$$

In obtaining Eqs. (27) and (28) we have made repeated use of the fact that $\int d \boldsymbol{\omega} \mathbf{e}(\boldsymbol{\omega}) \mathbf{e}(\boldsymbol{\omega})=(4 \pi / 3) \mathrm{U}$. By comparing Eqs. (19) and (28), we see that if the direct correlation function is of the form given in Eq. (26) then

$$
\mathbf{H}\left(\mathbf{r}, \mathbf{r}^{\prime}\right)=\mathbf{C}\left(\mathbf{r}, \mathbf{r}^{\prime}\right) \text {. }
$$

Thus if Eq. (26) is correct then $H\left(r, r^{\prime}\right)$ is, in fact, related to the direct correlation function in precisely the same way that $\mathbf{G}\left(\mathbf{r}, \mathbf{r}^{\prime}\right)$ is related to the total correlation function.

Now for nonpolar monatomic fluids there is considerable evidence that the direct correlation function becomes asymptotically equal to $-\beta \phi$ for large $\left|\mathbf{r}-\mathbf{r}^{\prime}\right|$, where $\phi$ is the intermolecular pair potential.6,10 This relation has also been said to hold for polar fluids. ${ }^{7}$ The validity of this relation will not be investigated here; we shall be concerned only with its consequences. Let us therefore assume that the quantity $c\left(\mathbf{r}, \boldsymbol{\omega} ; \mathbf{r}^{\prime}, \boldsymbol{\omega}^{\prime}\right)$ defined by Eq. (23) differs negligibly from $-\beta \phi(\mathbf{r}, \boldsymbol{\omega}$; $\left.\mathbf{r}^{\prime}, \boldsymbol{\omega}^{\prime}\right)$ if $\left|\mathbf{r}-\mathbf{r}^{\prime}\right|>\sigma$, where $\phi$ is the pair potential for the rigid-dipole model. ${ }^{2}$ But if $\left|r-r^{\prime}\right|>\sigma$ then $\phi$ differs negligibly from the dipole-dipole potential $\phi_{d}=$ $-\mu_{0}^{2} T\left(\mathbf{r}-\mathbf{r}^{\prime}\right): \mathbf{e}(\boldsymbol{\omega}) \mathbf{e}\left(\boldsymbol{\omega}^{\prime}\right)$. Our first assumption is therefore that

$$
\begin{aligned}
c\left(\mathbf{r}, \boldsymbol{\omega} ; \mathbf{r}^{\prime}, \boldsymbol{\omega}^{\prime}\right)=c_{0}\left(\mathbf{r}, \boldsymbol{\omega} ; \mathbf{r}^{\prime}, \boldsymbol{\omega}^{\prime}\right) & \\
& +\beta \mu_{0}{ }^{2} T_{\sigma}\left(\mathbf{r}-\mathbf{r}^{\prime}\right): \mathbf{e}(\boldsymbol{\omega}) \mathbf{e}\left(\boldsymbol{\omega}^{\prime}\right),
\end{aligned}
$$

where $c_{0}\left(\mathbf{r}, \boldsymbol{\omega} ; \mathbf{r}^{\prime}, \boldsymbol{\omega}^{\prime}\right) \approx 0$ if $\left|\mathbf{r}-\mathbf{r}^{\prime}\right|>\sigma$.

According to Eq. $(30), c\left(\mathbf{r}, \boldsymbol{\omega} ; \mathbf{r}^{\prime}, \boldsymbol{\omega}^{\prime}\right)$ is indeed of the form given in Eq. (26) if $\left|\mathbf{r}-\mathbf{r}^{\prime}\right|>\sigma$. But this is not sufficient to imply Eq. (29); if we want Eq. (29) to be valid then we must make the second assumption that $c_{0}\left(\mathbf{r}, \boldsymbol{\omega} ; \mathbf{r}^{\prime}, \boldsymbol{\omega}^{\prime}\right)$ is of the form given in Eq. (26). As mentioned in the Introduction, this assumption is almost certainly not rigorously correct, and the error incurred by adopting it is not easily estimated. Even if $c\left(\mathbf{r}, \boldsymbol{\omega} ; \mathbf{r}^{\prime}, \boldsymbol{\omega}^{\prime}\right)$ is in error only for small $\left|\mathbf{r}-\mathbf{r}^{\prime}\right|$, the convolution in Eq. (25) will propagate the error and cause $\mathbf{H}\left(\mathbf{r}, \mathbf{r}^{\prime}\right)$ to differ from $\mathbf{C}\left(\mathbf{r}, \mathbf{r}^{\prime}\right)$ for both small and large $\left|\mathbf{r}-\mathbf{r}^{\prime}\right|$.

We now go on to examine the consequences of the above two assumptions. Substitution of Eq. (30) into Eq. (24) yields

where

$$
\mathrm{C}\left(\mathrm{r}, \mathbf{r}^{\prime}\right)=\mathrm{C}_{0}\left(\mathbf{r}, \mathrm{r}^{\prime}\right)+\frac{1}{9} \mu_{0}^{2} \beta \mathbf{T}_{\sigma}\left(\mathbf{r}-\mathbf{r}^{\prime}\right),
$$

$$
\mathrm{C}_{0}\left(\mathbf{r}, \mathbf{r}^{\prime}\right)=(4 \pi)^{-2} \int d \boldsymbol{\omega} l \boldsymbol{\omega}^{\prime} c_{0}\left(\mathbf{r}, \boldsymbol{\omega} ; \mathbf{r}^{\prime}, \boldsymbol{\omega}^{\prime}\right) \mathbf{e}(\boldsymbol{\omega}) \mathbf{e}\left(\boldsymbol{\omega}^{\prime}\right) .
$$

Clearly $C_{0}\left(\mathbf{r}, \mathbf{r}^{\prime}\right) \approx 0$ if $\left|\mathbf{r}-\mathbf{r}^{\prime}\right|>\sigma$. Combining Eqs. (31), (29), and (20), we obtain

$$
\begin{aligned}
\mathrm{L}\left(\mathbf{r}, \mathbf{r}^{\prime}\right)=\left(\mu_{0}^{2} \beta \rho / 3\right)^{-1} \mathbf{U} \delta\left(\mathbf{r}-\mathbf{r}^{\prime}\right) & \\
& -\left(\mu_{0}^{2} \beta / 9\right)^{-1} \mathbf{C}_{0}\left(\mathbf{r}, \mathbf{r}^{\prime}\right)-\mathrm{T}_{\sigma}\left(\mathbf{r}-\mathbf{r}^{\prime}\right) .
\end{aligned}
$$

We therefore see that the above two assumptions imply that $L\left(\mathbf{r}, \mathbf{r}^{\prime}\right)$ is in fact of the form given in Eq. (11), with the short-ranged part $\mathbf{L}_{s}\left(\mathbf{r}, \mathbf{r}^{\prime}\right)$ given by

$$
\mathbf{L}_{s}\left(\mathbf{r}, \mathbf{r}^{\prime}\right)=\left(\mu_{0}^{2} \beta \rho / 3\right)^{-1} \bigcup \delta\left(\mathbf{r}-\mathbf{r}^{\prime}\right)-\left(\mu_{0}^{2} \beta / 9\right)^{-1} C_{0}\left(\mathbf{r}, \mathbf{r}^{\prime}\right) .
$$

We next combine Eqs. (34) and (13), obtaining

$$
\mathbf{A}(\mathbf{r})=\left(\mu_{0}^{2} \beta \rho / 3\right)^{-1} \mathbf{U}-\left(\mu_{0}^{2} \beta / 9\right)^{-1} \int_{\infty} d^{3} \mathbf{r}^{\prime} \mathbf{C}_{0}\left(\mathbf{r}, \mathbf{r}^{\prime}\right) .
$$

Now in order for $\epsilon$ to exist, the integral in Eq. (35) must be independent of $\mathbf{r}$ and proportional to $\mathrm{U}$, as discussed in Sec. II. In general, this will be the case only if $c_{0}\left(\mathbf{r}, \boldsymbol{\omega} ; \mathbf{r}^{\prime}, \boldsymbol{\omega}^{\prime}\right)$ depends only upon the relative positions and orientations of the two representative molecules involved. This constitutes a third assumption, which, however, is very reasonable in view of the physical interpretation of $c_{0}\left(\mathbf{r}, \boldsymbol{\omega} ; \mathbf{r}^{\prime}, \boldsymbol{\omega}^{\prime}\right)$ as the direct short-range correlation between two representative molecules. According to this third assumption, the functional dependence of $c_{0}\left(\mathbf{r}, \boldsymbol{\omega} ; \mathbf{r}^{\prime}, \boldsymbol{\omega}^{\prime}\right)$ may be expressed as $c_{0}\left(s, \boldsymbol{\Omega}, \boldsymbol{\Omega}^{\prime}\right)$, where $s=\left|\mathbf{r}-\mathbf{r}^{\prime}\right|$ and $\boldsymbol{\Omega}$ and $\boldsymbol{\Omega}^{\prime}$ denote orientation angles measured with respect to the intermolecular axis $\left(\mathbf{r}-\mathbf{r}^{\prime}\right)$. (In Ref. $2, \boldsymbol{\Omega}$ and $\boldsymbol{\Omega}^{\prime}$ were denoted by $\omega_{1}^{\prime}$ and $\omega_{2}^{\prime}$, respectively.) Our second and third assumptions may be combined into the single statement

$c_{0}\left(\mathbf{r}, \boldsymbol{\omega} ; \mathbf{r}^{\prime}, \boldsymbol{\omega}^{\prime}\right)=c_{s}\left(\left|\mathbf{r}-\mathbf{r}^{\prime}\right|\right)+\mathbf{F}\left(\mathbf{r}-\mathbf{r}^{\prime}\right): \mathbf{e}(\boldsymbol{\omega}) \mathbf{e}\left(\boldsymbol{\omega}^{\prime}\right)$,

where $c_{s}(|\mathbf{r}|) \approx 0$ and $\mathbf{F}(\mathbf{r}) \approx 0$ if $|\mathbf{r}|>\sigma$. The tensor function $F(\mathbf{r})$ is of course not completely arbitrary; it must have the property that the second term in Eq. (36) depends only upon $s, \boldsymbol{\Omega}$, and $\boldsymbol{\Omega}^{\prime}$.

Our third assumption above ensures that the integral in Eq. (35) is both independent of $\mathbf{r}$ and proportional 
to $U$. The constant of proportionality may be determined in exactly the same way that the constant $d(\mathbf{r})$ was determined in Ref. 2. The result is

where

$$
\int_{\infty} d^{3} \mathbf{r}^{\prime} \mathbf{C}_{0}\left(\mathbf{r}, \mathbf{r}^{\prime}\right)=(12 \pi)^{-1} \mathfrak{Q U},
$$

$$
Q=\int_{0}^{\infty} s^{2} d s \int d \boldsymbol{\Omega} d \mathbf{\Omega}^{\prime} c_{0}\left(s, \boldsymbol{\Omega}, \boldsymbol{\Omega}^{\prime}\right) \cos \gamma\left(\boldsymbol{\Omega}, \mathbf{\Omega}^{\prime}\right)
$$

and $\gamma\left(\boldsymbol{\Omega}, \boldsymbol{\Omega}^{\prime}\right)=\cos ^{-1}\left[\mathbf{e}(\boldsymbol{\omega}) \cdot \mathbf{e}\left(\boldsymbol{\omega}^{\prime}\right)\right]$. Since the dipoledipole potential is orientationally orthogonal to $\cos \gamma\left(\boldsymbol{\Omega}, \boldsymbol{\Omega}^{\prime}\right)$ [see Eq. (49) of Ref. 2], Eq. (30) implies that the value of $a$ will be unchanged if $c_{0}(s, \mathbf{\Omega}, \mathbf{\Omega})^{\prime}$ is replaced by $c\left(s, \mathbf{\Omega}, \boldsymbol{\Omega}^{\prime}\right)$ in Eq. (38). We may therefore rewrite $\mathrm{Eq}$. (38) as

$$
\alpha=\int_{0}^{\infty} s^{2} d s \int d \boldsymbol{\Omega} d \mathbf{\Omega}^{\prime} c\left(s, \boldsymbol{\Omega}, \boldsymbol{\Omega}^{\prime}\right) \cos \gamma\left(\boldsymbol{\Omega}, \boldsymbol{\Omega}^{\prime}\right) .
$$

Combining Eqs. (37) and (35) and comparing the result to Eq. (14), we obtain

$$
A=\left(\mu_{0}^{2} \beta \rho / 3\right)^{-1}[1-(\rho / 4 \pi) Q] .
$$

According to Eq. (16), therefore,

$$
(3 / 4 \pi)[(\epsilon-1) /(\epsilon+2)]=\frac{1}{3} \mu_{0}^{2} \beta \rho[1-(\rho / 4 \pi) Q]^{-1},
$$

where $Q$ is given by either Eq. (39) or Eq. (38).

This completes the development described in the Introduction. Our basic assumptions are summarized by Eqs. (30) and (36). We have seen that if these assumptions are satisfied then the dielectric constant exists, in which case it is given explicitly in terms of the direct correlation function by Eqs. (41) and (39). This expression for $\epsilon$ appears to be new.

Equation (41) may serve as the basis for an expansion of the Clausius-Mossotti function

$$
(\epsilon-1) /[(\epsilon+2) \rho]
$$

in powers of $\rho$. It is a simple matter to verify that the first two terms in the expansion are identical to those obtained in Ref. 2. This provides a limited check on Eq. (41) and our development.

We emphasize again that deviations from Eq. (36) may be expected to cause $\mathbf{H}\left(\mathbf{r}, \mathbf{r}^{\prime}\right)$ to differ from $\mathbf{C}\left(\mathbf{r}, \mathbf{r}^{\prime}\right)$ at both short and long range. If there were only a shortrange difference then the main effect would be a change in the value of $\epsilon$. Any long-range difference between $\mathbf{H}\left(\mathbf{r}, \mathbf{r}^{\prime}\right)$ and $\mathbf{C}\left(\mathbf{r}, \mathbf{r}^{\prime}\right)$, however, would imply that $\epsilon$ no longer rigorously exists, since even if this difference is proportional to $T_{\sigma}\left(\mathbf{r}-\mathbf{r}^{\prime}\right)$ the long-range part of $L\left(\mathbf{r}, \mathbf{r}^{\prime}\right)$ will no longer completely cancel with the $T_{\sigma}\left(\mathbf{r}-\mathbf{r}^{\prime}\right)$ appearing in Eq. (10).

A question of considerable interest is whether $\mathrm{Eq}$. (41) for $\epsilon$ is equivalent to the well-known Kirkwood equation..$^{12}$ Since the Kirkwood equation emerges from the treatment of Nienhuis and Deutch, this question is closely related to that of the relationship between our work and theirs. A direct demonstration that Eq. (41) and the Kirkwood equation are equivalent appears to be a nontrivial task. However, it is easy to give an indirect proof that these two equations are indeed equivalent within the framework of conditions (a) and (b) [Eqs. (30) and (36)]. If conditions (a) and (b) are satisfied then we have seen that $\epsilon$ exists and is given by Eq. (41). But the Kirkwood equation is a logical consequence of the assumption that $\epsilon$ exists. Therefore conditions (a) and (b) imply the Kirkwood equation as well. The question of whether Eq. (41) remains equivalent to the Kirkwood equation when conditions (a) and (b) are not satisfied is of less interest, since as discussed above $\epsilon$ does not then appear to exist.

\section{THE MEAN SPHERICAL MODEL OF WERTHEIM}

Wertheim has recently published a study of the mean spherical model (MSM) for polar fluids. ${ }^{13}$ The MSM includes our condition (a) (with $\sigma$ replaced by a hardsphere diameter $R$ ) as part of its definition, and also defines $\rho^{(2)}$ to be zero if $\left|\mathbf{r}-\mathbf{r}^{\prime}\right|<R$. Wertheim shows that this model is exactly soluble. He first considers the infinite-volume case, and then the finite-volume case for the special case of a spherical volume. He does not investigate the existence of $\epsilon$, but under the assumption that $\epsilon$ exists he obtains an explicit expression for it in closed form. This expression is obtained by considering the familiar case of a spherical sample in a uniform external electric field.

It is not difficult to show that our expression for $\epsilon$ [Eqs. (41) and (39)] reduces, when specialized to the MSM, to Wertheim's expression. For this purpose, equations in Wertheim's article will be identified by a "W"; e.g., Eq. (W63). If we substitute Eq. (W12) for the direct correlation function into Eq. (39), we obtain

$$
Q=\frac{1}{3}(4 \pi)^{2} \int_{0}^{R} s^{2} d s c_{\Delta}(s),
$$

where Eq. (W30) has been used. Equation (W41), together with the fact that $\hat{c}_{\Delta}(r)=c_{\Delta}(r)$, implies that $c_{\Delta}(r)=2 K\left[c_{+}(r)-c_{-}(r)\right]$. Combining this relation with Eqs. (W45) and substituting the result into Eq. (42), we obtain

$$
\begin{aligned}
Q & =\frac{1}{3}(4 \pi)^{2}(2 K) \int_{0}^{R} s^{2} d s\left[c_{\delta}(s, 2 K \rho)-c_{s}(s,-K \rho)\right] \\
& =(4 \pi / 3 \rho)[3-q(2 \xi)-2 q(-\xi)],
\end{aligned}
$$

where Eq. (W50) has been used. Substituting Eq. (43) in to Eq. (41), we obtain

$$
(\epsilon-1) /(\epsilon+2)=3 y[q(2 \xi)+2 q(-\xi)]^{-1},
$$

where $y=4 \pi \mu_{0}^{2} \beta \rho / 9$. If we now eliminate $y$ from Eq. (44) by means of $\mathrm{Eq}$. (W51), we obtain precisely Eq. (W63). This agreement provides a simultaneous check on both our work and Wertheim's.

It is also noteworthy that Wertheim's expression for 
the direct correlation function, Eq. (W12), is of the form of our Eq. (26). This means that our condition (b) is rigorously satisfied for the MSM, and since condition (a) is satisfied by definition we see that the dielectric constant is rigorously well defined for the MSM. The MSM therefore acquires the distinction of being the only nontrivial model of a polar fluid for which $\epsilon$ is known rigorously to exist. We re-emphasize, however, that condition (b) is not in general expected to be satisfied; its validity for the MSM must for the present be regarded as a feature peculiar to this particular model. ${ }^{14}$

\section{CONCLUDING REMARKS}

We have seen that it is convenient to investigate the existence of $\epsilon$ by focusing attention on the kernel $\mathbf{L}\left(\mathbf{r}, \mathbf{r}^{\prime}\right)$ inverse to the kernel $\mathbf{K}\left(\mathbf{r}, \mathbf{r}^{\prime}\right)$, since if $\epsilon$ exists the former exhibits a much simpler behavior than the latter. This approach leads one naturally to inquire into the behavior of the direct correlation function in polar fluids. It is found that sufficient conditions for the existence of $\epsilon$ can be simply expressed in terms of the short- and long-range behavior of the direct correlation function. The condition on the long-range behavior is intuitively quite plausible, but that on the short-range behavior is probably valid only to a certain degree of approximation. These results lend a great deal of new intuition and insight into the question of the existence of $\epsilon$ for polar fluids.

The present context provides a good example of how the direct correlation function may be a useful concept even though it is not short ranged in comparison to the total correlation function. The direct correlation function is useful not because it is short ranged but because it is simple. Since in the present case the system is finite and the direct correlation function is long ranged, the convolution in Eq. (23) transforms the simple behavior of the direct correlation function into a complicated shape-dependent behavior for the total correlation function or $\rho^{(2)}$. The use of the direct correlation function allows one to see how this complicated behavior, which at first appears somewhat mysterious, may arise in a simple manner.

It should be emphasized, however, that the definition of the direct correlation function is intuitively motivated, and one has no real assurance that this quantity rigorously possesses the simple physical interpretation we would like it to. The basic idea behind the concept is simple and has a strong intuitive appeal, namely that the total correlation between two molecules may be regarded as the sum of a direct and an indirect effect, the indirect effect being transmitted by chains of direct effects between intermediate molecules. All possible such chains must be summed over. The definition of the direct correlation function is based upon the tacit supposition that a chain of direct correlations involving more than two molecules can be expressed as a product (actually a convolution) of direct pair correlations. (This is reminiscent of a Markoffian assumption, usually encountered with time as the random variable.) The sum over chains may then formally be carried out to yield Eq. (23). Logically, of course, Eq. (23) is simply the definition of the direct correlation function, and thus involves no assumption whatever. The above discussion is intended only to emphasize that care must be taken in attributing to the direct correlation function properties which are not strictly implied by its definition.

\section{ACKNOWLEDGMENT}

I am grateful to Professor George Stell for a number of thoughtful comments and suggestions, and for making his preliminary results available prior to publication.

* Present address: Applied Theory, Inc., 1010 Westwood Boulevard, Los Angeles, California 90024.

${ }^{1}$ J. D. Ramshaw, Ph.D. thesis, Massachusetts Institute of Technology, Cambridge, Mass., 1970 (unpublished).

${ }^{2}$ J. D. Ramshaw, J. Chem. Phys. 55, 1763 (1971).

3 J. D. Ramshaw, "Existence of the Dielectric Constant in Nonpolar Fluids," Physica (to be published).

${ }^{4}$ Most of the important papers on dielectric theory are included in the references cited in Refs. 2 and 3. A reference which was previously overlooked is: D. J. Vezzetti and J. B. Keller, J. Math. Phys. 8, 1861 (1967). Although these authors use an infinite sample (of unspecified shape) and do not discuss the existence of $\epsilon$, their work may bear indirectly on this question for nonpolar fluids.

5 A. N. Kaufman and K. M. Watson, Phys. Fluids 4, 931 (1961)

${ }^{6}$ See, for example, M. D. Johnson, P. Hutchinson, and N. H. March, Proc. Roy. Soc. (London) A282, 283 (1964); J. E. Enderby, T. Gaskell, and N. H. March, Proc. Phys. Soc. (London) 85,217 (1965); P. A. Egelstaff, An Introduction to the Liquid State (Academic, London, 1967), p. 58.

${ }^{7}$ F. M. Kuni, Phys. Letters 26A, 305 (1968).

${ }^{8} \mathrm{G}$. Nienhuis and J. M. Deutch, J. Chem. Phys. 55, 4213 (1971)

${ }_{9}^{\mathrm{G}}$. S. Rushbrooke, in Physics of Simple Liquids (NorthHolland, Amsterdam, 1968), p. 25.

${ }^{10} \mathrm{~J}$. L. Lebowitz, G. Stell, and S. Baer, J. Math. Phys. 6, $1282(1965)$.

11 The advantage of inverting the relation between $P(r)$ and $\mathrm{E}_{0}(\mathbf{r})$ was first realized, in another connection, by $\mathrm{J}$. Yvon, Compt. Rend. 202, 35 (1936).

12 J. G. Kirkwood, J. Chem. Phys. 7, 911 (1939)

${ }_{13}$ M. S. Wertheim, J. Chem. Phys. 55, 4291 (1971)

$14 \mathrm{G}$. Stell (private communication) has obtained some preliminary results which indicate that condition (b) may be satisfied under more general circumstances, provided one continues to impose the condition that $\rho^{(2)}=0$ for $\left|r_{1}-r_{2}\right|<\sigma$. He also finds that the leading asymptotic correction to condition (a) is a term of order $\left|r_{1}-r_{2}\right|^{-6}$. One is relieved to see that this correction is indeed short ranged in comparison to the dipole-dipole potential. These results provide still further evidence that our basic formulation is a useful and appropriate one. We understand that Professor Stell intends to report his results in more detail in a forthcoming publication. 